Check for updates

Cite this: RSC Adv., 2017, 7, 31549

Received 24th February 2017

Accepted 25th May 2017

DOI: 10.1039/c7ra02313h

rsc.li/rsc-advances

\section{Preparation and adsorption behaviors of sodium alginate-based adsorbent-immobilized $\beta$ - cyclodextrin and graphene oxide}

\author{
Yanchen Wu, † Houjuan Qi,† Cai Shi, Rongxiu Ma, Shouxin Liu and Zhanhua Huang (DD * \\ Highly water soluble graphene oxide (GO) was synthesized via a modified Hummers method. A sodium \\ alginate (SA)-based adsorbent-immobilized $\beta$-cyclodextrin ( $\beta-C D$ ) and GO gel (SCGG) with excellent \\ regeneration ability were prepared. The adsorptive properties of methylene blue (MB) on the SCGG \\ adsorbent were studied. The results showed that when the dosage of the adsorbent was $1.0 \mathrm{~g}, \mathrm{pH}$ of the \\ solution was 7, temperature was $35{ }^{\circ} \mathrm{C}$, initial concentration of $\mathrm{MB}$ was $50 \mathrm{mg} \mathrm{L}^{-1}$, and adsorption time \\ was $180 \mathrm{~min}$, the removal rate of $\mathrm{MB}$ was $84.98 \%$ and the adsorption capacity was $133.24 \mathrm{mg} \mathrm{g}^{-1}$. The \\ isotherms and kinetics of adsorption were investigated to reveal that the equilibrium adsorption and \\ kinetics were well-described by the Freundlich model and pseudo-second-order kinetics, respectively. \\ The thermodynamic parameters showed that the adsorption process was spontaneous and endothermic \\ in nature. The mechanical property of the SCGG was improved by the addition of GO. The regeneration \\ removal rate of $\mathrm{MB}$ was more than $82 \%$ after six recycles.
}

\section{Introduction}

As is well-known, dyes are widely used in the textile, papermaking, printing, and other industries. Dyes offer convenience to consumers; however, the use of dyes can cause serious harm to the environment due to the indiscriminate discharge of dye wastewater, ${ }^{1,2}$ and the deterioration of the environment will affect the human health. How to control the content of dyes in wastewater is a matter of great concern among researchers. ${ }^{3}$ Many physical and chemical methods such as adsorption, physical precipitation and flocculation, coagulation, chemical oxidation and reduction, biological treatment, and photocatalytic degradation ${ }^{4}$ have been used to remove dyes from wastewater. Among these, adsorption has been developed to deal with dye wastewater problem because this method is faster, cost-effective, and environmentally friendly; furthermore, the design and operation of adsorption process is comparatively easier and it does not result in secondary pollution. The MB dye is a heteroaromatic compound that is used in industrial applications, and it can affect the quality of water. Various adsorbents, such as rice husk, ${ }^{5}$ garlic peel, ${ }^{6}$ conducing polymers, ${ }^{7}$ carbon nanotubes, ${ }^{8}$ bamboo activated carbon, ${ }^{9}$ cedar sawdust, and crushed brick, ${ }^{10}$ have been used for the adsorption of MB. However, some of these adsorbents show limitations in terms of

Key Laboratory of Bio-based Material Science and Technology of the Ministry of Education, Northeast Forestry University, Harbin 150040, China. E-mail: nefuhzh@ nefu.edu.cn

$\dagger$ Co-first authors. recycling; therefore, an adsorbent with good adsorption capacity and regeneration performance is highly desirable.

A number of adsorbents based on biomass resources have been researched. ${ }^{11-14}$ Among these, the SA-based adsorbent is particularly interesting. SA was derived from brown algae and bacterial biosynthesis. It comprises sequences of $\mathrm{M}$ (M-blocks) and $\mathrm{G}$ (G-blocks) residues interspersed with MG sequences (MG-blocks). ${ }^{15} \mathrm{SA}$ is biodegradable, biocompatible, renewable, and nontoxic. The guluronic acid of SA can be cross-linked via divalent or trivalent cations (such as $\mathrm{Ca}^{2+}$ ) to obtain an egg-box structure, ${ }^{16,17}$ forming a gel. However, the mechanical property of the gel obtained from SA was poor, which limits its regeneration. In order to improve the mechanical property of SA gel, some researchers have explored. Jie et al. prepared a carboxyl multi-walled carbon nanotube/calcium alginate composite membrane, and the results showed that the tensile strength of the membrane was improved..$^{18}$ Chang et al. fabricated hydrogels by blending cellulose and SA solution for the improvement of the mechanical property. ${ }^{19} \mathrm{GO}$ was also used to enhance the mechanical property via a wet spinning method. ${ }^{20} \mathrm{GO}$ is oxygenated derivative of graphene and possess many excellent properties. GO surfaces and edges contain rich oxygencontaining functional groups including the carboxyl, hydroxyl, and epoxy groups. Moreover, GO exhibits good hydrophilicity and dispersion due to these oxygen-containing functional groups. ${ }^{21}$ The large specific surface area and rich oxygencontaining functional groups of GO can provide abundant sites for adsorption. Moreover, when GO is used as a filler, its unique mechanical property can enhance the strength of the material. The outer rim of $\beta-\mathrm{CD}$ is hydrophilic and its inner 
cavity is hydrophobic. Moreover, $\beta$-CD possesses a special structure and rich functional groups and can increase the number of oxygen-containing functional groups and adsorption active sites. In addition, $\beta$-CD can improve the dispersion of gel balls.

The objective of this study was to obtain a high mechanical property SA-based adsorbent with excellent adsorption and regeneration ability. To improve the mechanical property and the dispersion of the gel beads, GO and $\beta$-CD were immobilized on an SA matrix. SCGG was prepared via an ion exchange method with SA, GO, and $\beta$-CD. To explore the potential application of SCGG in dye removal, MB dye was chosen to test the adsorption behaviors of SCGG. The adsorption isotherms, adsorption kinetics, and thermodynamic performance were studied via batch experiments. This study can provide methods for the development of high-performance biological adsorbents. The method for the preparation of the SCGG adsorbent was simple. Furthermore, SCGG possesses good adsorption properties such as shorter equilibrium time, faster adsorption rate, and higher adsorption capacity. Moreover, the mechanical properties of SCGG were improved, and the regeneration ability of SCGG reached at least 6 times due to the addition of GO.

\section{Materials and methods}

\subsection{Materials}

Graphite with an average particle size of $30 \mu \mathrm{m}$ was purchased from Sinopharm Chemical Reagent Co., Ltd. (Shanghai, China). MB cationic dye was obtained from YongDa Chemical Reagent Co., Ltd. (Tianjin, China). SA biopolymer with a viscosity of 1.05-1.15 Pa s was obtained from GuangFu Fine Chemical Research Institute (Tianjin, China). $\beta$-CD was purchased from Kermel Chemical Reagent Co., Ltd. (Tianjin, China). All other reagents used were of analytical grade or better and used as received. Herein, $0.1 \mathrm{M} \mathrm{NaOH}$ and $0.1 \mathrm{M} \mathrm{HCl}$ were used to adjust the $\mathrm{pH}$ of the solutions. Deionized water was used to prepare all the dye solutions.

\subsection{Preparation of GO}

GO was synthesized from natural graphite powder via a modified Hummers method..$^{22,23}$ In a typical procedure, graphite powder was placed in an $80{ }^{\circ} \mathrm{C}$ solution of concentrated $\mathrm{H}_{2} \mathrm{SO}_{4}$ with $\mathrm{K}_{2} \mathrm{~S}_{2} \mathrm{O}_{8}$ and $\mathrm{P}_{2} \mathrm{O}_{5}$ for $5 \mathrm{~h}$. The mixture was washed with deionized water to remove the residual acid, and the mixture was filtered and dried. The obtained powder was oxidized via the Hummers method. ${ }^{24}$ The obtained powder was placed in $\mathrm{H}_{2} \mathrm{SO}_{4}(98 \%, 60 \mathrm{~mL})$ in a round-bottom flask under the stirring condition. Then, $\mathrm{KMnO}_{4}$ was added while the temperature of the mixture was maintained below $5{ }^{\circ} \mathrm{C}$. After this, the temperature was increased to $35{ }^{\circ} \mathrm{C}$ for $2 \mathrm{~h}$; then, the mixture was diluted with de-ionized water $(92 \mathrm{~mL})$, and the temperature was increased to $70{ }^{\circ} \mathrm{C}$ for $0.5 \mathrm{~h}$. After this, deionized water $(100$ $\mathrm{mL})$ and $\mathrm{H}_{2} \mathrm{O}_{2}(20 \mathrm{~mL})$ were added to the mixture, and the mixture was filtered and washed with $\mathrm{HCl}$ aqueous and deionized water to remove metal ions and acid. The resulting filter cake was purified via dialysis to remove the remaining metal species. Finally, the suspension was ultrasonically treated for $2 \mathrm{~h}$ and centrifuged to remove the unexfoliated GO particles. The GO suspension was thus obtained.

\subsection{Preparation of SCGG}

The GO suspension was diluted using $100 \mathrm{~mL}$ deionized water via ultrasonic dispersion for $2 \mathrm{~h}$. SA and $\beta$-CD were added to the above mentioned solution with the mass ratio of $1: 1$. The mixed solution was continuously stirred until a homogeneous solution was formed. Then, the homogeneous solution was left undisturbed to remove the air bubbles. After this, the homogeneous solution was dropped into the $5 \mathrm{wt} \%$ solution of $\mathrm{CaCl}_{2}$ using a syringe. The beads were left in the $\mathrm{CaCl}_{2}$ solution for $12 \mathrm{~h}$ and washed three times with deionized water to obtain the stable gel beads (SCGG).

\subsection{Adsorption and regeneration experiments}

MB solutions were prepared from the corresponding standard stock solutions by diluting with deionized water to different concentrations. MB solution was taken in Erlenmeyer flasks with fixed volume (50 mL). SCGG was added to the MB solutions in different amounts. The Erlenmeyer flasks were placed in a water-bathing constant temperature vibrator. The upper layer liquid was analyzed at a wavelength of $664 \mathrm{~nm}$ using a UVvisible spectrophotometer after a certain time. The removal rate $(R)(1)$ and adsorption capacity $\left(q_{\mathrm{e}}\right)(2)$ were calculated as follows:

$$
\begin{aligned}
& R=\frac{C_{0}-C_{\mathrm{e}}}{C_{0}} \times 100 \% \\
& q_{\mathrm{e}}=\frac{\left(C_{0}-C_{\mathrm{e}}\right) \times V}{m}
\end{aligned}
$$

where $C_{0}\left(\mathrm{mg} \mathrm{L}^{-1}\right)$ and $C_{\mathrm{e}}\left(\mathrm{mg} \mathrm{L}^{-1}\right)$ are the initial and equilibrium concentrations of the $\mathrm{MB}$ solutions, respectively; $m(\mathrm{~g})$ is the weight of the SCGG used; and $V(\mathrm{~L})$ is the volume of the MB solution.

The SCGG was desorbed using $0.1 \mathrm{~mol} \mathrm{~L}^{-1} \mathrm{HCl}$ solution after saturated adsorption of MB. The Erlenmeyer flask that contained $0.1 \mathrm{~mol} \mathrm{~L}^{-1} \mathrm{HCl}$ and saturated SCGG was placed in a water-bathing constant temperature vibrator for $3 \mathrm{~h}$ to complete desorption. Then, SCGG was filtered and washed three times to reach neutrality prior to the next adsorption experiment.

\subsection{Deformation rate}

The different weight of the balancing weight was put on gel balls to change their diameters, and a Vernier caliper was used to measure the average diameters.

\subsection{Characterization}

Atomic force microscopy (AFM, PicoPlus, Molecular Imaging, USA) was used to analyze the thickness of the samples in the tapping mode at a scanning rate of $0.5 \mathrm{~Hz}$. Fourier transform infrared (FTIR) spectrum was obtained using a Fourier transform infrared spectrometer (iS10, Nicolet, USA). The spectra 
were acquired in the wavenumber range from 4000 to $400 \mathrm{~cm}^{-1}$. $\mathrm{X}$-ray diffraction (XRD) patterns were obtained using an advance diffractometer (D/MAX2200, Rigaku, Japan) with $\mathrm{Cu} \mathrm{K} \alpha$ radiation $(\lambda=1.5418 \AA)$ operating at $40 \mathrm{kV}$ and $30 \mathrm{~mA}$. Samples were scanned in the range $5^{\circ}<2 \theta<60^{\circ}$ with a scan interval of $0.02^{\circ}$. The morphology and structure of the samples were characterized via a scanning electron microscope (SEM, QUANTA200, FEI, Holland), and the samples were treated with spray gold. Frequently, the samples were covered using a vacuum cover system for the evaporation of metals. UV-vis spectra were acquired using a spectrophotometer (TU-1900, Beijing Purkinje General, China) in the scan range between 200 and $800 \mathrm{~nm}$. A transmission electron microscope (JEM-2100, JEOL, Japan) was used to study the morphology of the samples at an accelerating voltage of $200 \mathrm{KV}$. Thermogravimetric analysis (TGA, Q50, TA instrument, USA) was used to detect the thermal stability under a $\mathrm{N}_{2}$ atmosphere at the heating rate of $10{ }^{\circ} \mathrm{C} \mathrm{min}^{-1}$ from $25^{\circ} \mathrm{C}$ to $600{ }^{\circ} \mathrm{C}$.

\section{Results and discussion}

\subsection{Sample characteristics}

The information about the size and thickness of the GO sheets was obtained via AFM. As shown in Fig. 1(a), the GO sheets exist as micrometer-sized planes, and the thickness of the GO flakes is very uniform, ranging from 1 to $2 \mathrm{~nm}$, suggesting the complete exfoliation of GO sheets down to individual or bilayer sheets. The TEM image in Fig. 1(f) shows a good dispersion of GO nanosheets with some crinkles on the surface. The GO
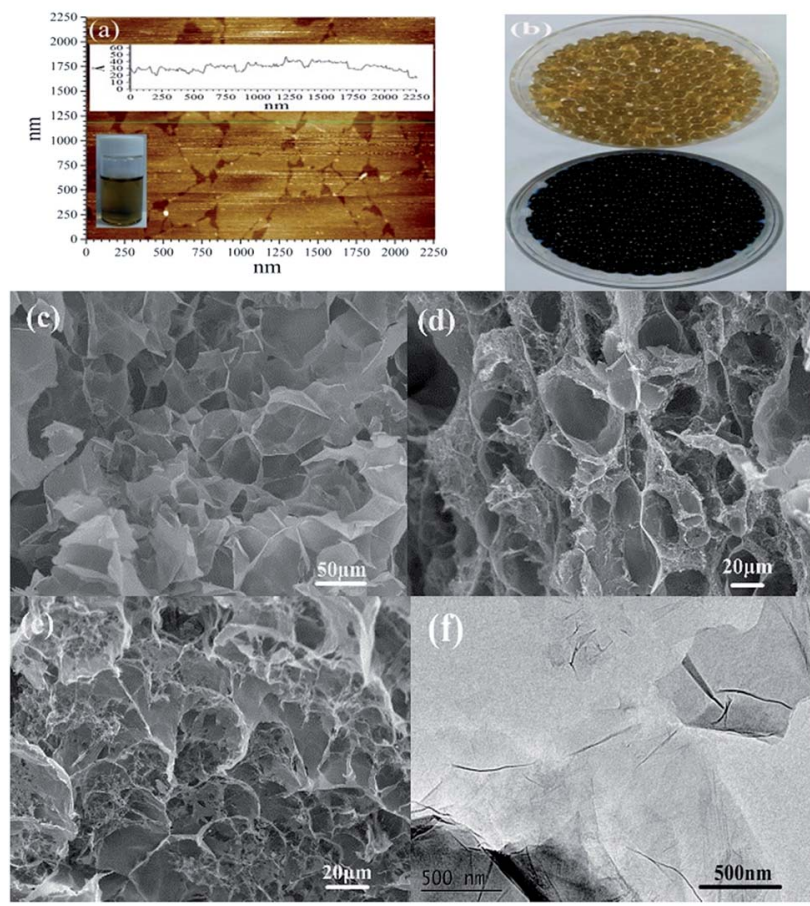

Fig. 1 The AFM image of GO (a), images obtained before and after adsorption (b), SEM images (c-e) of SCGG and TEM image of GO (f). solution exhibited excellent water solubility and could retain its homogeneous state for a long time.

The image of MB adsorption on SCGG before and after is shown in Fig. 1(b). As seen from Fig. 1(b), SCGG has a uniform size and is faint yellow in color because of the presence of GO. However, after the adsorption of MB, SCGG turned dark blueblack.

The internal morphology of the SCGG is shown in Fig. 1(c-e). As shown in the figure, a honeycomb-like three-dimensional net structure can be clearly seen, and the pores of the SCGG are different. The structure not only increases the specific surface area of SCGG, but also increases the adsorption capacity of the active adsorptive sites, which is beneficial for the adsorption of the MB.

The FT-IR spectra of GO, SA, $\beta$-CD, SCGG- 1 (before adsorption), and SCGG-2 (after adsorption) are shown in Fig. 2(a). The broad adsorption band at $3312 \mathrm{~cm}^{-1}$ is ascribed to the $-\mathrm{OH}$ stretching vibration of the hydroxyl group. ${ }^{25}$ The band at 2925 $\mathrm{cm}^{-1}$ is assigned to the antisymmetric stretching vibrations of the methylene group. The peak appearing at $1731 \mathrm{~cm}^{-1}$ is associated with the $-\mathrm{C}=\mathrm{O}$ stretching vibrations of the carbonyl and carboxylic groups. The absorption bands around $1600 \mathrm{~cm}^{-1}$ can be attributed to the asymmetric stretching vibrations of the COO- group. ${ }^{26}$ The peak at $1418 \mathrm{~cm}^{-1}$ was assigned to $\mathrm{C}-\mathrm{OH}$. Moreover, the $\mathrm{C}-\mathrm{O}-\mathrm{C}$ and $\mathrm{C}-\mathrm{O}$ stretching vibration peaks appeared at $1223 \mathrm{~cm}^{-1}$ and $1023 \mathrm{~cm}^{-1}$, respectively. The presence of various oxygenous functional groups indicated that graphite was successfully oxidized. The abundant oxygenous functional groups make GO sheets strongly hydrophilic, which improve their solubility in water. These spectral results show that SCGG not only contains SA, $\beta$-CD, and GO, but also demonstrate the presence of composite reactions. In the spectra of SCGG-2, the peak at $1486 \mathrm{~cm}^{-1}$ was assigned to $\mathrm{C}=\mathrm{N}$; the stretching vibration peak of $\mathrm{C}-\mathrm{N}$ is located at $881 \mathrm{~cm}^{-1}$, and the stretching vibration peaks at $730-900 \mathrm{~cm}^{-1}$ can be attributed to the skeleton of benzene. Based on these results, it can be concluded that MB adsorption on SCGG has occurred.
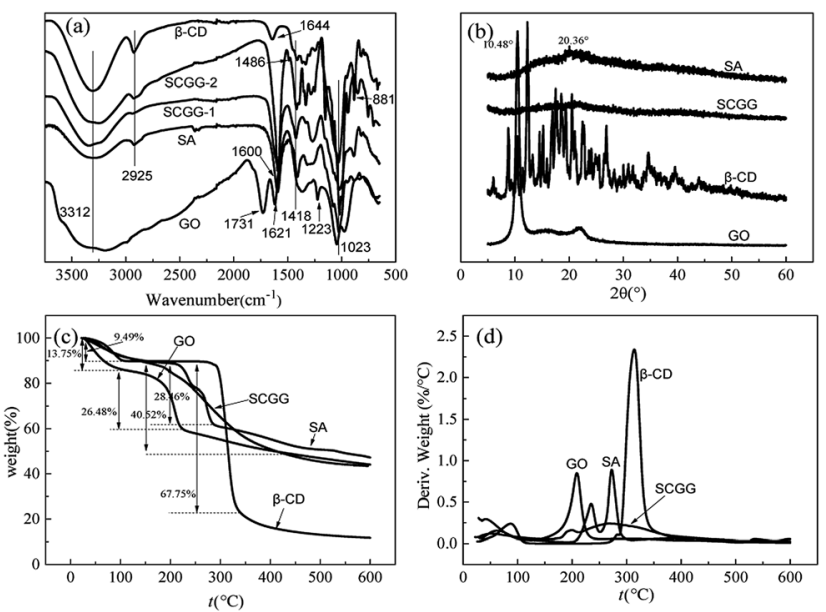

Fig. 2 FT-IR spectra (a), XRD patterns (b), TGA (c) and DTG (d) curves of GO, SA, $\beta-C D$, and SCGG. 
XRD patterns of GO, SA, $\beta$-CD, and SCGG are in Fig. $2(\mathrm{~b})$. The GO pattern showed an intense and sharp peak at $10.48^{\circ}$. According to the Bragg equation, ${ }^{27}$ this peak corresponded to a $d$-spacing of $0.8441 \mathrm{~nm}$, which confirms the synthesis of GO. ${ }^{28}$ In the XRD spectrum of SA, the broad peak at $20.36^{\circ}$ demonstrated the amorphous structure of SA. SA was usually semicrystalline due to the strong interaction between alginate chains through intermolecular hydrogen bonding. ${ }^{29}$ The XRD pattern of $\beta$-CD showed multiple diffraction peaks, which indicated that $\beta$-CD had a non-crystal structure. The SCGG pattern was similar to the SA pattern. This may be due to the low GO content in the SCGG. Because of the low content of GO in SCGG, the XRD spectrum of SCGG did not show a significant GO diffraction peak; moreover, more information was obtained, which indicated that the SCGG had an amorphous structure.

To determine the thermal stability, the mass-temperature relation was studied via TGA. TGA and DTG curves of GO, SA, $\beta$ $\mathrm{CD}$, and SCGG are shown in Fig. 2(c and d). In the case of GO and $\beta$-CD, the first weight loss stage between 25 and $100{ }^{\circ} \mathrm{C}$ was associated with the evaporation of water adsorbed on the surface. With the increasing temperature, a major weight lost was observed from 100 to $225{ }^{\circ} \mathrm{C}$, attributed to the thermal decompositions of oxygenous functional groups of GO. ${ }^{30}$ The weight loss of $\beta$-CD reached $67.75 \%$ from 100 to $340^{\circ} \mathrm{C}$; this can be attributed to the decomposition of oxygenous functional groups and the hexatomic ring chains fractured..$^{31}$ The TGA curve trends of SA and SCGG were similar in the temperature range from 25 and $150{ }^{\circ} \mathrm{C}$, which were assigned to adsorbed water on the surface. The temperature of the second weight loss stage reached 290 and $430^{\circ} \mathrm{C}$ for SA and SCGG, respectively. The second degradation temperature was increased. The difference in temperature may be attributed to the egg-box structure formed due to ion exchange of $\mathrm{Ca}^{2+}$. Thus, SCGG had high thermal stability.

\subsection{Adsorption and regeneration studies}

To choose a suitable dosage of adsorbent, different dosages of SCGG were added to $50 \mathrm{~mL}$ of $50 \mathrm{mg} \mathrm{L}^{-1} \mathrm{MB}$ solutions. The results are shown in Fig. 3(a). The adsorption capacity for MB dyes on SCGG decreased from $214.32 \mathrm{mg} \mathrm{g}^{-1}$ to $133.24 \mathrm{mg} \mathrm{g}^{-1}$ with the increase in dosage from 0.5 to $1.0 \mathrm{~g}$. The adsorption capacity decreased with the further increase in the amount of SCGG, whereas the removal rate increased with the increase in the amount of SCGG. This could be attributed to the increased surface area of the absorbent and availability of more active adsorptive sites with the increase in the amount of SCGG. ${ }^{32}$ However, a further increase in the amount of the absorbent caused a decrease in the utilization rate of the SCGG active adsorption sites. ${ }^{33}$ Thus, the $1.0 \mathrm{~g}$ amount of SCGG was considered for the next study.

$\mathrm{H}^{+}$concentration in solution is an important parameter that influences the adsorption behavior of dyes in an aqueous solution. It affects the surface charge of the adsorbent and the structure of the dyes. ${ }^{34}$ The effects of the solution $\mathrm{pH}$ (from 4 to 10) on the adsorption are presented in Fig. 3(b). The adsorption capacity for MB dye on SCGG increased from $118.64 \mathrm{mg} \mathrm{g}^{-1}$ to
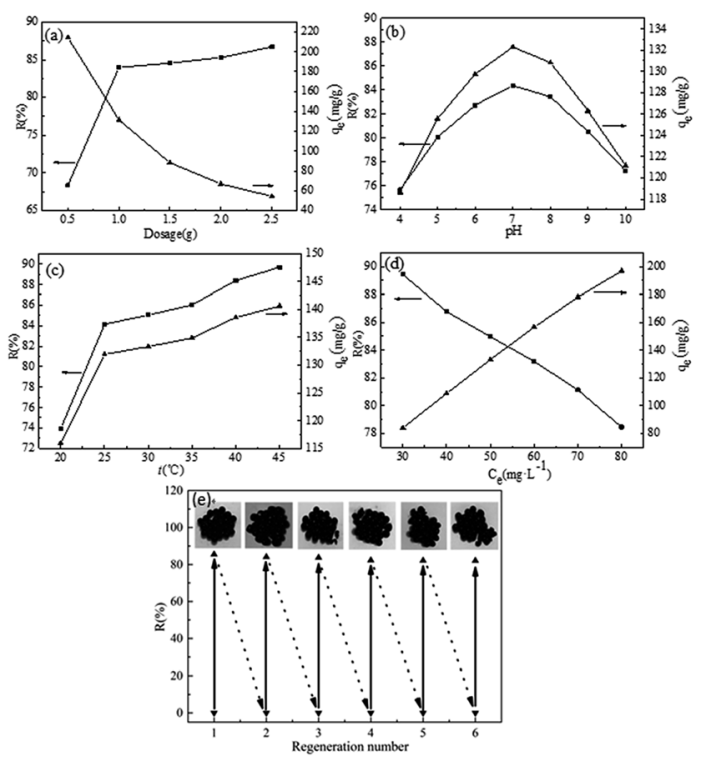

Fig. 3 Effects of adsorbent dose (a), solution pH (b), temperature (c), and initial MB concentration (d) on adsorption and the regeneration of SCGG (inset is the image of SCGG after adsorption) (e).

$133.24 \mathrm{mg} \mathrm{g}^{-1}$ as the $\mathrm{pH}$ approached 7.0. Moreover, the removal rate of MB dye on SCGG increased from $75.67 \%$ to $84.98 \%$. Thereafter, with the increase of solution $\mathrm{pH}$, the removal rate and adsorption capacity showed a decreasing trend. As a cationic dye, MB exists in the aqueous solution in the form of cationic groups $\left(\mathrm{MB}^{+}\right)$. This could be explained on the basis of the $\mathrm{H}^{+}$concentration. When the $\mathrm{pH}$ was low, $\mathrm{H}^{+}$competed with MB for the active adsorptive sites on the SCGG. ${ }^{35}$ With the increase of $\mathrm{pH}$, the decrease of $\mathrm{H}^{+}$in solution was beneficial for the adsorption of MB on SCGG. The removal rate and adsorption capacity of SCGG for MB showed a decreasing tendency when the $\mathrm{pH}$ was more than 7 . The mutual attraction between $\mathrm{MB}$ and $\mathrm{OH}^{-}$affected the removal rate and adsorption capacity of SCGG for MB.

Temperature affects the diffusion capacity of the dye molecules and the viscosity of the solution. The diffusion capacity of the dye molecules was enhanced, and the solution viscosity decreased with the increase in temperature. Furthermore, the equilibrium capacity of the adsorbent for a particular adsorbate can be modified by changing the temperature. Effects of temperature on the adsorption are shown in Fig. 3(c). The removal rate and adsorption capacity of SCGG for MB showed an increasing trend with an increase in temperature. The abovementioned results indicated that the adsorption process was endothermic in nature. This could be attributed to the following facts: (I) as the temperature increased, the diffusion capacity of the dye molecules enhanced, and the diffusion of MB into the active adsorptive sites became much easier ${ }^{36}$ and (II) the viscosity of the solution decreased with an increase in temperature.

MB adsorption is significantly influenced by the initial concentration of $\mathrm{MB}$ in the aqueous solution. The effect of the initial concentration of $\mathrm{MB}$ ranging from 30 to $80 \mathrm{mg} \mathrm{L}^{-1}$ on the 
SCGG adsorption was investigated, and the results are presented in Fig. 3(d). The adsorption capacity of MB dye gradually increased when the concentration increased. On the contrary, the removal rate of the MB dye gradually decreased when the concentration increased. Owing to the number of MB molecules was less to the active adsorptive sites when the concentration is low. ${ }^{37}$ Therefore, the removal rate was high. The effective chance of collision between MB and SCGG was increased and the mass transfer resistance decreased with the increase in the initial concentration of $\mathrm{MB} .^{38}$ Therefore, the adsorption capacity of SCGG for MB gradually increased. However, due to the saturation of SCGG active adsorption sites, low removal rate of MB was observed.

The regeneration ability of an adsorbent is an important factor to evaluate the practical application of the adsorbent. The breakage rate of SCGG in the adsorption-desorption eqn (3) is expressed as follow:

$$
B=\frac{N_{\mathrm{b}}}{N_{\mathrm{a}}} \times 100 \%
$$

where $N_{\mathrm{b}}$ is the breakage number of SCGG and $N_{\mathrm{a}}$ is the total number of SCGG.

SCGG-0\%, SCGG-5\%, and SCGG-10\% represent SCGG containing 0,5 , and $10 \%$ GO, respectively. From Table 1, it was observed that the deformation rate of SCGG decreased with the increase in the GO content. This result suggested that the mechanical property of SCGG increased with the increase in the content of GO.

The cycles of adsorption-desorption experiments were carried out, as shown in Fig. 3(e). The removal rate of MB decreased for each new cycle after desorption for six cycles. After six cycles, the removal rate of MB on SCGG was still above $82 \%$ and the adsorption capacity was more than $130.68 \mathrm{mg} \mathrm{g}^{-1}$, which demonstrated that SCGG could be effectively regenerated and reused for the adsorption of $\mathrm{MB}$ with good adsorption performance. The breakage rates of SCGG- $0 \%$ in the regeneration recycle were $0,4,8,16,24$, and $32 \%$. The breakage rates of
Table 2 Comparison of adsorption capacities and regeneration number of various adsorbents for MB removal

\begin{tabular}{|c|c|c|c|}
\hline Adsorbent & $q_{\mathrm{e}}\left(\mathrm{mg} \mathrm{g}^{-1}\right)$ & $\begin{array}{l}\text { Regeneration } \\
\text { number }\end{array}$ & Sources \\
\hline $\begin{array}{l}\text { Modified ball clay and } \\
\text { chitosan composite }\end{array}$ & 26.93 & 5 & 39 \\
\hline Potato leaves powder & 52.60 & 3 & 40 \\
\hline Magnetic chitosan and GO & 95.16 & 4 & 41 \\
\hline $\begin{array}{l}\text { Starch-humic } \\
\text { composite hydrogel }\end{array}$ & 110.00 & 5 & 42 \\
\hline GO-SA & 123.10 & - & 43 \\
\hline Citrus limetta peel & 227.30 & - & 44 \\
\hline SCGG & 132.09 & 6 & $\begin{array}{l}\text { This } \\
\text { work }\end{array}$ \\
\hline
\end{tabular}

SCGG in the regeneration recycle were $0,0,4,12,16$, and $20 \%$ because the spherical structure of SCGG is gradually destroyed during the desorption process of shock. The mechanical property of the SCGG was enhanced due to the addition of GO; although the spherical structure SCGG was damaged during the process of desorption, it did not affect the adsorption properties. The regeneration removal rates of $\mathrm{MB}$ were $84.98,84.14$, $83.76,82.32,82.15$, and $82.08 \%$ in six cycles. The adsorption capacity and regeneration number of MB on SCGG was considerable as compared to those of other biological adsorbents reported in the literature, as shown in Table 2. Scheme 1 illustrates the probable reaction between SCGG and MB. ${ }^{45}$ The SA and $\beta$-CD were biodegradable, which have a good prospect.

\subsection{Study of the adsorption isotherms}

The interaction model between SCGG and MB could be described using the adsorption isotherms. Adsorption isotherms were obtained at different temperatures. The adsorption isotherm reflected the linear relationship between the adsorption capacity and equilibrium concentration of the

Table 1 Mechanical property and breakage rate of SCGG with different contents of GO

\begin{tabular}{|c|c|c|c|c|c|c|}
\hline \multirow[b]{2}{*}{ SCGG-0\% } & \multirow{2}{*}{$\begin{array}{l}\text { Weight }(\mathrm{g}) \\
0\end{array}$} & \multirow{2}{*}{$\begin{array}{l}\text { Length }(\mathrm{cm}) \\
8.58\end{array}$} & \multirow{2}{*}{$\begin{array}{l}\text { Diameter }(\mathrm{cm}) \\
0.2860\end{array}$} & \multirow{2}{*}{$\begin{array}{l}\text { Deformation rate (\%) } \\
0\end{array}$} & \multicolumn{2}{|c|}{$\begin{array}{l}\text { Breakage rate } \\
\text { in } 5 \text { cycles (\%) }\end{array}$} \\
\hline & & & & & 1 & 0 \\
\hline & 20 & 8.94 & 0.2980 & 4.1958 & 3 & 8 \\
\hline & 50 & 9.13 & 0.3043 & 6.4103 & 4 & 16 \\
\hline & 100 & 9.25 & 0.3083 & 7.8089 & 5 & 24 \\
\hline & 20 & 8.85 & 0.2950 & 2.4306 & 3 & 4 \\
\hline & 50 & 8.96 & 0.2987 & 3.7037 & 4 & 8 \\
\hline & 100 & 9.04 & 0.3013 & 4.6296 & 5 & 16 \\
\hline \multirow[t]{3}{*}{ SCGG-10\% } & 0 & 8.05 & 0.2683 & 0 & 1 & 0 \\
\hline & 10 & 8.11 & 0.2703 & 0.7453 & 2 & 0 \\
\hline & 20 & 8.14 & 0.2713 & 1.1180 & 3 & 0 \\
\hline
\end{tabular}




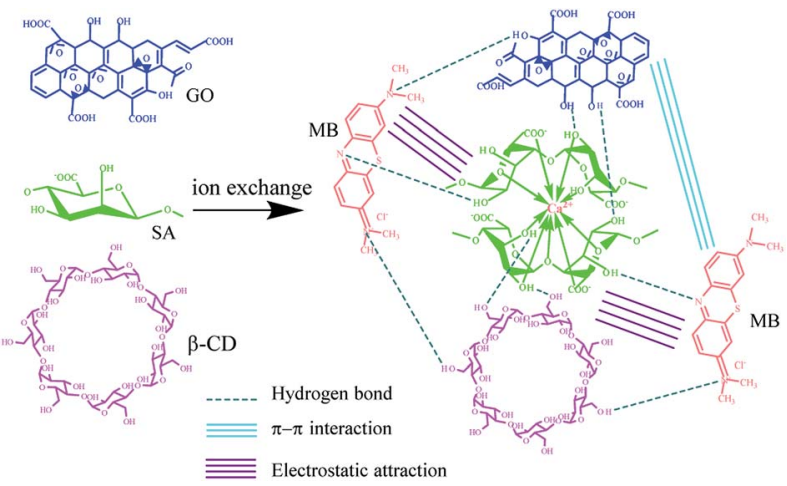

Scheme 1 Schematic of the probable reaction between SCGG and MB.

solution at a certain temperature. The adsorption isotherm model can be further used to study the mechanism of adsorption. The commonly used model, including Langmuir $(4)^{46}$ and Freundlich $(5)^{47}$ model, is as follows:

$$
\begin{aligned}
& \frac{C_{\mathrm{e}}}{q_{\mathrm{e}}}=\frac{C_{\mathrm{e}}}{q_{\max }}+\frac{1}{q_{\max } k_{\mathrm{L}}} \\
& \ln q_{\mathrm{e}}=\ln k_{\mathrm{F}}+\frac{1}{n} \ln C_{\mathrm{e}}
\end{aligned}
$$

where $C_{\mathrm{e}}\left(\mathrm{mg} \mathrm{L}^{-1}\right)$ is the equilibrium concentration of the MB solution; $q_{\mathrm{e}}\left(\mathrm{mg} \mathrm{g}^{-1}\right)$ is the amount of MB adsorbed on the absorbent; $q_{\max }\left(\mathrm{mg} \mathrm{g}^{-1}\right)$ is the maximum amount of $\mathrm{MB}$ adsorbed on the absorbent; $k_{\mathrm{L}}\left(\mathrm{L} \mathrm{mg}^{-1}\right)$ is the Langmuir equilibrium adsorption constant; $k_{\mathrm{F}}$ is the Freundlich characteristic adsorption constant; and $n$ is the constant.

The experimental data were analyzed using the Langmuir model and Freundlich model, as shown in Fig. 4(a-c). The fitting parameters are listed in Table 3. As observed in the from Table 3, the correlation coefficient of the Freundlich model $\left(R^{2}>\right.$ $0.99)$ was greater than that of the Langmuir model $\left(R^{2}>0.98\right)$ at the same temperature, which indicated that the experimental data fitted well with the Freundlich model. Furthermore, the essential feature of the Langmuir isotherm model is a separation factor $R_{\mathrm{L}}(6)$, belonging to a dimensionless constant, which is defined by

$$
R_{\mathrm{L}}=\frac{1}{1+C_{0} k_{\mathrm{L}}}
$$

where $C_{0}$ is the initial MB concentration and $k_{\mathrm{L}}$ is the Langmuir equilibrium constant. The values of $R_{\mathrm{L}}$ indicates the tendency of the adsorption process, which can be unfavorable $\left(R_{\mathrm{L}}>1\right)$, linear $\left(R_{\mathrm{L}}=1\right)$, favorable $\left(0<R_{\mathrm{L}}<1\right)$, irreversible $\left(R_{\mathrm{L}}=0\right)$, and unfavorable $\left(R_{\mathrm{L}}<0\right)$. In this study, all the $R_{\mathrm{L}}$ values were 0.1491 , 0.1514 , and 0.1559 corresponding to 25,35 , and $45^{\circ} \mathrm{C}$, respectively, which indicated that the adsorption of MB on SCGG was favorable. ${ }^{48}$ The values of $n$ in the range of 1-10 suggested favorable adsorption for MB on the SCGG. ${ }^{49}$ This may be attributed to the large specific surface area of GO and the rich functional groups present in SCGG.
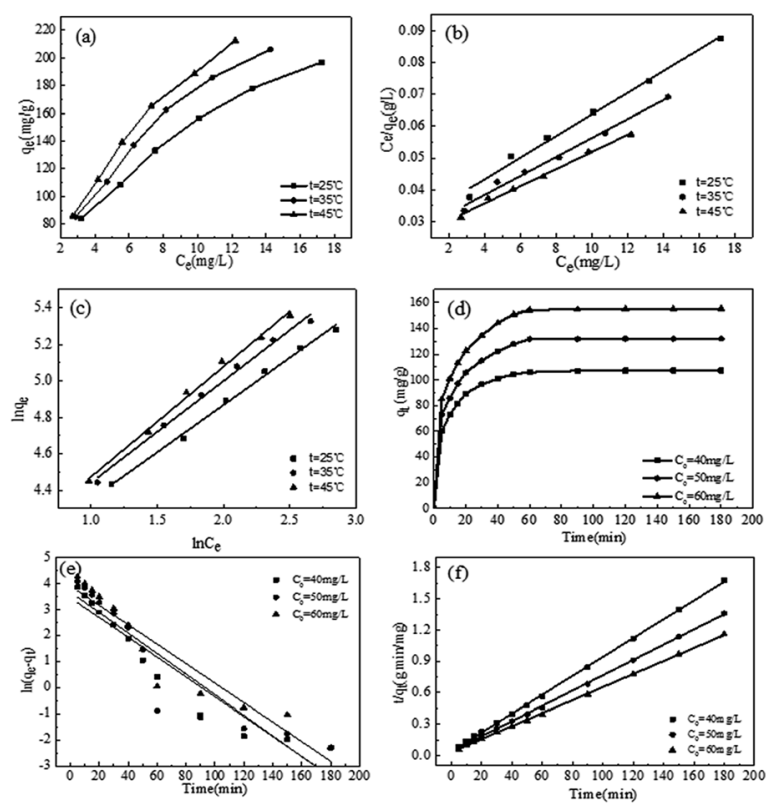

Fig. 4 Adsorption isotherms of MB on SCGG (a), adsorption data obtained and fitted with different models: Langmuir (b), Freundlich (c) at different temperatures, kinetic curves of different initial concentrations of $M B(d)$, and adsorption kinetics data of MB onto SCGG fitted with the pseudo-first-order model (e) and the pseudo-second-order model (f).

\subsection{Study of the adsorption kinetics}

To investigate the mechanism and kinetics for the adsorption of MB on SCGG, adsorption experiments were carried out using three different concentrations of $\mathrm{MB}\left(20,30,40 \mathrm{mg} \mathrm{L}^{-1}\right)$, and the results are shown in Fig. 4(d-f). As observed from Fig. 4(d), with the extension of adsorption time, the adsorption of $\mathrm{MB}$ on SCGG was a fast process in the initial $20 \mathrm{~min}$, the adsorption rate slowed down between 20 and $60 \mathrm{~min}$, and the adsorption process reached a balance at $120 \mathrm{~min}$. This can be attributed to the high concentration of $\mathrm{MB}$ in the solution and more active adsorption sites in the initial stage of adsorption; moreover, the mass transfer driving force was large. Therefore, the adsorption rate of MB on SCGG was fast. As adsorption progressed, the concentration of MB decreased and the active adsorption sites were gradually saturated; thus, the adsorption rate became slow. As the MB molecules on the surface of SCGG entered the internal areas of SCGG via diffusion, the adsorption capacity slowly increased and eventually reached equilibrium. Therefore, the adsorption time required for the adsorption capacity to reach equilibrium was about $180 \mathrm{~min}$.

Adsorption kinetics is used to study the linear relationship between the adsorption capacity and time. The commonly used models include the pseudo-first-order kinetic eqn $(7)^{50}$ and the pseudo-second-order kinetic eqn (8). ${ }^{4}$ They are calculated using the following equations

$$
\begin{gathered}
\ln \left(q_{\mathrm{e}}-q_{t}\right)=\ln q_{\mathrm{e}}-k_{1} t \\
\frac{t}{q_{t}}=\frac{1}{k_{2} q_{\mathrm{e}}{ }^{2}}+\frac{t}{q_{\mathrm{e}}}
\end{gathered}
$$


Table 3 The isothermal adsorption parameters and thermodynamic parameters of MB adsorption on SCGG at different temperatures

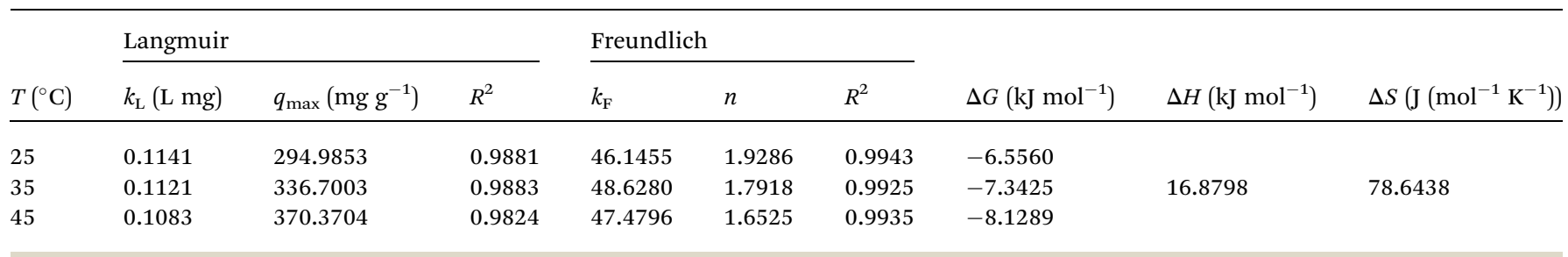

Table 4 Kinetic parameters of different initial concentrations of $\mathrm{MB}^{a}$

\begin{tabular}{|c|c|c|c|c|c|c|c|c|}
\hline$C_{0}\left(\mathrm{mg} \mathrm{L}^{-1}\right)$ & \multicolumn{4}{|c|}{ Pseudo-first-order kinetic equation } & \multicolumn{4}{|c|}{ Pseudo-second-order kinetic equation } \\
\hline 50 & 0.0394 & 39.1703 & 133.2428 & 0.8455 & 0.0015 & 136.6120 & 133.2428 & 0.9895 \\
\hline 60 & 0.0373 & 49.3861 & 155.1953 & 0.9014 & 0.0013 & 160.5136 & 155.1953 & 0.9876 \\
\hline
\end{tabular}

where $q_{\mathrm{e}}\left(\mathrm{mg} \mathrm{g}^{-1}\right)$ is the equilibrium adsorption capacity; $q_{t}(\mathrm{mg}$ $\left.\mathrm{g}^{-1}\right)$ is the adsorption capacity at $t ; k_{1}\left(\mathrm{~min}^{-1}\right)$ is the pseudofirst-order kinetic rate constant; and $k_{2}\left(\mathrm{~g}\left(\mathrm{mg}^{-1} \mathrm{~min}^{-1}\right)\right)$ is the pseudo-second-order kinetic constant. The kinetic equation and adsorption data are listed in Table 4.

The experimental data were fitted with the pseudo-first-order kinetic equation and the pseudo-second-order kinetic equation. The fitting results are shown in Table 4. As observed from Table 4, when the initial concentration of $\mathrm{MB}$ was $40 \mathrm{mg} \mathrm{L}^{-1}$, there were significant differences between the equilibrium adsorption values calculated by the pseudo-first-order kinetic equation (31.3044 $\mathrm{mg} \mathrm{g}^{-1}$ ) and those calculated via the experiment $\left(107.3619 \mathrm{mg} \mathrm{g}^{-1}\right)$. On the contrary, the equilibrium adsorption capacity calculated by the pseudo-second-order kinetic equation (110.4972 $\mathrm{mg} \mathrm{g}^{-1}$ ) was closer to the experimental value $\left(107.3619 \mathrm{mg} \mathrm{g}^{-1}\right)$. The results of the other two concentrations were consistent with this rule. Furthermore, the correlation coefficients of the pseudo-second-order kinetic equation $\left(R^{2}>\right.$ $0.98)$ were higher than those of the pseudo-first-order kinetic equation $\left(R^{2}>0.84\right)$. Therefore, the experimental data were well represented by the pseudo-second-order kinetic equation.

\subsection{Study of the adsorption thermodynamic}

To further study the influence of temperature on the adsorption of $\mathrm{MB}$ and explore the thermodynamic changes of the adsorption process, adsorption experiments were carried out at 298, 308 , and $318 \mathrm{~K}$. The adsorption equilibrium constant (9) and thermodynamic eqn (10) and (11) are expressed as follows:

$$
\begin{gathered}
K_{\mathrm{d}}=\frac{q_{\mathrm{e}}}{C_{\mathrm{e}}} \\
\ln K_{\mathrm{d}}=-\frac{\Delta H}{R T}+\frac{\Delta S}{R}
\end{gathered}
$$

$$
\Delta G=\Delta H-T \Delta S
$$

where $K_{\mathrm{d}}\left(\mathrm{L} \mathrm{g}^{-1}\right)$ is the distribution coefficient; $q_{\mathrm{e}}\left(\mathrm{mg} \mathrm{g}^{-1}\right)$ is the adsorption capacity at equilibrium; $C_{\mathrm{e}}\left(\mathrm{mg} \mathrm{L}^{-1}\right)$ is the concentration of the MB solution at equilibrium; $R\left(8.314 \mathrm{~J}_{\left(\mathrm{mol}^{-1}\right.}\right.$ $\left.\left.\mathrm{K}^{-1}\right)\right)$ is the ideal gas constant; $\Delta H\left(\mathrm{~kJ} \mathrm{~mol}^{-1}\right)$ is the standard change in enthalpy; $\Delta S\left(\mathrm{~J}\left(\mathrm{~mol}^{-1} \mathrm{~K}^{-1}\right)\right)$ is the standard change in entropy; and $\Delta G\left(\mathrm{~kJ} \mathrm{~mol}^{-1}\right)$ is the Gibbs free energy change in a given process.

Moreover, thermodynamic parameters were calculated according to eqn (9)-(11), and the results are shown in Table 3. $\Delta G$ values were calculated to be $-6.5560,-7.3425$, and -8.1289 $\mathrm{kJ} \mathrm{mol}^{-1}$ at 298,308 , and $318 \mathrm{~K}$, respectively. Negative $\Delta G$ values indicated spontaneous adsorption. ${ }^{51}$ Furthermore, $\Delta G$ values decreases with the increase in temperature, which indicate that better adsorption performance can be obtained when the temperature is higher. The positive values of $\Delta H$ confirmed the endothermic nature of the overall adsorption process. ${ }^{52}$ Finally, the positive value of $\Delta S$ suggested an increased randomness at the solid-liquid interface during the adsorption of $\mathrm{MB}$ on SCGG. ${ }^{53}$

\section{Conclusion}

In the present study, highly water soluble GO was synthetized via a modified Hummers method. A novel SA-based adsorbent (SCGG) was successfully prepared, and the removal of MB in an aqueous solution was studied using SCGG. The equilibrium data were well-modeled by the Freundlich isotherm model. The kinetic study showed that the adsorption process followed the pseudo-second-order kinetic model. The thermodynamic data indicated that the adsorption process was spontaneous and endothermic in nature. Moreover, the mechanical property of SCGG was improved, the deformation rate was decreased, and the breakage rate was decreased due to the addition of GO. The 
removal rate of $\mathrm{MB}$ was significant after six cycles. Based on the above mentioned experimental results and the advantages, such as cost-effectiveness, biodegradability, ease of operation, and high efficiency, of SCGG, it can be concluded that SCGG has great potential applications in industrial wastewater treatment and environmental protection.

\section{Acknowledgements}

This study was funded by the National Natural Science Foundation of China (No. 31670592), the Central University Basic Scientific Research Project of China (No. 2572017EB03), and the Research Funds for the Returned People of Heilongjiang Province (No. LC2016008).

\section{Notes and references}

1 M. Bhaumik, R. McCrindle and A. Maity, Chem. Eng. J., 2013, 228, 506-515.

2 H. Mittal, V. Kumar, Saruchi and S. S. Ray, Int. J. Biol. Macromol., 2016, 89, 1-11.

3 V. K. Gupta and Suhas, J. Environ. Manage., 2009, 90, 23132342.

4 N. K. Goel, V. Kumar, N. Misra and L. Varshney, Carbohydr. Polym., 2015, 132, 444-451.

5 Y. Li, Q. Du, T. Liu, Y. Qi, P. Zhang, Z. Wang and Y. Xia, Appl. Surf. Sci., 2011, 257, 10621-10627.

6 B. H. Hameed and A. A. Ahmad, J. Hazard. Mater., 2009, 164, 870-875.

7 D. Mahanta, G. Madras, S. Radhakrishnan and S. Patil, $J$. Phys. Chem. B, 2009, 113, 2293-2299.

8 Y. Yao, F. Xu, M. Chen, Z. Xu and Z. Zhu, Bioresour. Technol., 2010, 101, 3040-3046.

9 B. H. Hameed, A. T. M. Din and A. L. Ahmad, J. Hazard. Mater., 2007, 141, 819-825.

10 O. Hamdaoui, J. Hazard. Mater., 2006, 135, 264-273.

11 E. Alver, M. Bulut, A. Ü. Metin and H. Çiftçi, Spectrochim. Acta, Part A, 2017, 171, 132-138.

12 N. E. Mousa, C. M. Simonescu, R.-E. Pătescu, C. Onose, C. Tardei, D. C. Culiță, O. Oprea, D. Patroi and V. Lavric, React. Funct. Polym., 2016, 109, 137-150.

13 K. Shweta and H. Jha, Biotechnol. Rep., 2015, 7, 95-106.

14 X. Zhu, L. Bao, Y. Wei, J. Ma and Y. Kong, Int. J. Biol. Macromol., 2016, 91, 409-415.

15 S. N. Pawar and K. J. Edgar, Biomaterials, 2012, 33, 32793305.

16 L. Sun and B. Fugetsu, Chem. Eng. J., 2014, 240, 565-573.

17 S. Zhang, F. Xu, Y. Wang, W. Zhang, X. Peng and F. Pepe, Chem. Eng. J., 2013, 234, 33-42.

18 G. Jie, Z. Kongyin, Z. Xinxin, C. Zhijiang, C. Min, C. Tian and W. Junfu, Mater. Lett., 2015, 157, 112-115.

19 C. Chang, B. Duan and L. Zhang, Polymer, 2009, 50, 54675473.

20 Y. He, N. Zhang, Q. Gong, H. Qiu, W. Wang, Y. Liu and J. Gao, Carbohydr. Polym., 2012, 88, 1100-1108.

21 W. Peng, H. Li, Y. Liu and S. Song, J. Mol. Liq., 2016, 221, 8287.
22 Y. Pan, T. Wu, H. Bao and L. Li, Carbohydr. Polym., 2011, 83, 1908-1915.

23 M.-j. Li, C.-m. Liu, Y.-b. Xie, H.-b. Cao, H. Zhao and Y. Zhang, Carbon, 2014, 66, 302-311.

24 W. S. Hummers and R. E. Offeman, J. Am. Chem. Soc., 1958, 80, 1339.

25 Y. Zhang, Y. Liu, X. Wang, Z. Sun, J. Ma, T. Wu, F. Xing and J. Gao, Carbohydr. Polym., 2014, 101, 392-400.

26 M. M. Lakouraj, F. Mojerlou and E. N. Zare, Carbohydr. Polym., 2014, 106, 34-41.

27 W. H. Bragg and W. L. Bragg, Proc. R. Soc. London, Ser. A, 1913, 88, 428-438.

28 T.-T. Wu and J.-M. Ting, Surf. Coat. Technol., 2013, 231, 487491.

29 S. Thakur, S. Pandey and O. A. Arotiba, Carbohydr. Polym., 2016, 153, 34-46.

30 G. Jiang, Z. Lin, C. Chen, L. Zhu, Q. Chang, N. Wang, W. Wei and H. Tang, Carbon, 2011, 49, 2693-2701.

31 A. Mahmood, M. Ahmad, R. M. Sarfraz and M. U. Minhas, J. Drug Delivery Sci. Technol., 2016, 36, 75-88.

32 H. Ren, Z. Gao, D. Wu, J. Jiang, Y. Sun and C. Luo, Carbohydr. Polym., 2016, 137, 402-409.

33 X. Sun, J. H. Chen, Z. Su, Y. Huang and X. Dong, Chem. Eng. J., 2016, 290, 1-11.

34 A. Sigdel, J. Park, H. Kwak and P.-K. Park, J. Ind. Eng. Chem., 2016, 35, 277-286.

35 R. Ahmad, J. Hazard. Mater., 2009, 171, 767-773.

36 V. Srivastava, C. H. Weng, V. K. Singh and Y. C. Sharma, J. Chem. Eng. Data, 2011, 56, 1414-1422.

37 C. Cheng, L. Ma, J. Ren, L. Li, G. Zhang, Q. Yang and C. Zhao, Chem. Eng. J., 2011, 171, 1132-1142.

38 A. Benhouria, M. A. Islam, H. Zaghouane-Boudiaf, M. Boutahala and B. H. Hameed, Chem. Eng. J., 2015, 270, 621-630.

39 M. Auta and B. H. Hameed, Chem. Eng. J., 2014, 237, 352361.

40 N. Gupta, A. K. Kushwaha and M. C. Chattopadhyaya, Arabian J. Chem., 2016, 9(1), S707-S716.

41 L. Fan, C. Luo, X. Li, F. Lu, H. Qiu and M. Sun, J. Hazard. Mater., 2012, 215-216, 272-279.

42 R. Chen, Y. Zhang, L. Shen, X. Wang, J. Chen, A. Ma and W. Jiang, Chem. Eng. J., 2015, 268, 348-355.

43 T. Ma, P. R. Chang, P. Zheng, F. Zhao and X. Ma, Chem. Eng. J., 2014, 240, 595-600.

44 S. Shakoor and A. Nasar, J. Taiwan Inst. Chem. Eng., 2016, 66, 154-163.

45 M. Heidarizad and S. S. Şengör, J. Mol. Liq., 2016, 224, 607617.

46 G. Zhang, L. Yi, H. Deng and P. Sun, J. Environ. Sci., 2014, 26, 1203-1211.

47 J.-H. Deng, X.-R. Zhang, G.-M. Zeng, J.-L. Gong, Q.-Y. Niu and J. Liang, Chem. Eng. J., 2013, 226, 189-200.

48 L.-g. Yan, K. Yang, R.-r. Shan, T. Yan, J. Wei, S.-j. Yu, H.-q. Yu and B. Du, J. Colloid Interface Sci., 2015, 448, 508-516.

49 Y. Li, Q. Du, T. Liu, J. Sun, Y. Wang, S. Wu, Z. Wang, Y. Xia and L. Xia, Carbohydr. Polym., 2013, 95, 501-507. 
50 P. Sharma, B. K. Saikia and M. R. Das, Colloids Surf., A, 2014, 457, 125-133.

51 A. N. Fernandes, C. A. P. Almeida, N. A. Debacher and M. M. d. S. Sierra, J. Mol. Struct., 2010, 982, 62-65.
52 L. Ai, C. Zhang and Z. Chen, J. Hazard. Mater., 2011, 192, 1515-1524.

53 S. Shrestha, G. Son, S. H. Lee and T. G. Lee, Chemosphere, 2013, 92, 1053-1061. 